\title{
La investigación biográfico narrativa: significados y tendencias en la indagación de la identidad profesional docente*
}

\author{
The Biographical \\ Narrative \\ Research: Meaning \\ and Tendencies \\ Searching \\ for Teachers' \\ Professional Identity
}

\author{
Pesquisa \\ biográfica \\ narrativa: \\ significados e \\ tendências na \\ investigação \\ da identidade \\ profissional dos \\ professores
}

Ibeth del Rosario Morales Escobar** https://orcid.org/0000-0001-9359-4992

María Alejandra Taborda Caro*** https://orcid.org/0000-0002-9170-661X

El presente artículo de revisión hace parte de una de las fases de la investigación titulada: La configuración identitaria de profesores memorables que enseñan didáctica en la formación complementaria. Un estudio biográfico narrativo en docentes de las Escuelas Normales Superiores de Córdoba, realizado en el marco del Doctorado en Educación de la Universidad Nacional de Rosario (Argentina).

** Magíster en Educación de la Universidad de Córdoba. Docente de tiempo completo de la Facultad de Educación y Ciencias Humanas de esta Universidad. Grupo de Investigación Sociedades, Imaginarios y Comunicación.

Correo electrónico: ibethmoralese@correo.unicordoba.edu.co

*** Doctora en Educación de la Universidad Pedagógica Nacional. Coordinadora de la Maestría en Ciencias Sociales de la Universidad de Córdoba. Grupo de Investigación Sociedades, Imaginarios y Comunicación.

Correo electrónico: socialescolombia@gmail.com

Primer semestre de $2021 \cdot$ pp. 171-182 Segunda época

N. ${ }^{0}=0$ 


\title{
Resumen
}

En este artículo de revisión se presentan los resultados de una exploración sistemática de literatura cuyo objetivo general fue reconocer los alcances y principales procesos metodológicos de la investigación narrativa, además de contar con información para determinar vacíos en este campo de investigación. Para ello, se seleccionaron investigaciones centradas en las categorías de identidad docente y método biográfico narrativo. El método para el análisis de la información fue el estudio de metasíntesis, empleado para integrar y generar nuevas interpretaciones de los resultados de investigaciones cualitativas. Esto, mediante la aplicación de fichas de revisión y la realización de análisis documental. Los resultados indican que la investigación biográfico narrativa ha permitido desarrollar conocimiento sobre la formación docente; la vida y la experiencia profesional de los profesores; sobre cómo las condiciones sociopolíticas, familiares y educativas influyen en el desarrollo personal y profesional; acerca de las concepciones sobre la buena enseñanza, la identidad docente, el saber pedagógico, los rasgos de los docentes memorables y la riqueza de la investigación narrativa.

\section{Palabras clave}

método de investigación; identidad; profesión docente

\begin{abstract}
This following review article presents the results of a systematic exploration of the literature whose general objective was to recognize the main methodological processes of narrative research, as well as to have information to determine gaps in this field of research. For this, research focused on the categories of teaching identity and narrative biographical method were selected. The method of information analysis was the study of metasynthesis, used to integrate and generate new interpretations of qualitative research results. This is done by applying review guides and performing documentary analysis. The results indicate that narrative biographical research enabled the development of knowledge about teacher education; teachers' lives and professional experience; how sociopolitical, family and educational conditions influence personal and professional development; on conceptions of good teaching, teaching identity, pedagogical knowledge, traits of memorable teachers and the richness of narrative research.

\section{Keywords}

research method; identity; teaching profession

Este artigo de revisão apresenta os resultados de uma exploração sistemática da literatura cujo objetivo geral era reconhecer os principais processos metodológicos da pesquisa narrativa, bem como ter informações para determinar lacunas neste campo de pesquisa. Para isso, foram selecionadas pesquisas focadas nas categorias de identidade docente e método biográfico narrativo. 0 método de análise da informação foi o estudo da metassíntese, utilizado para integrar e gerar novas interpretações dos resultados qualitativos da pesquisa. Isso é feito aplicando guias de revisão e realizando análises documentais. Os resultados indicam que a pesquisa biográfica narrativa possibilitou o desenvolvimento do conhecimento sobre a formação docente; vida dos professores e experiência profissional; como as condições sociopolíticas, familiares e educacionais influenciam o desenvolvimento pessoal e profissional; sobre concepções de bom ensino, identidade docente, conhecimento pedagógico, traços de professores memoráveis e a riqueza da pesquisa narrativa.
\end{abstract}

Abstract

\section{Resumo}

\section{Palavras chave}

método de investigação; identidade; profissão docente 


\section{Introducción}

En la agenda investigativa relacionada con la comprensión de la identidad docente y la búsqueda de estrategias y técnicas para conocer, reconstruir y poner a circular en el ámbito epistémico, su pensamiento y saber pedagógico, la investigación biográfico narrativa se ha convertido en aliada inseparable para aquellos que han volcado su mirada hacia la forma como el contacto con el contexto sociocultural, las trayectorias de vida, la educación y las formas de educar de los maestros. Lo cual ha permitido llevar a cabo experiencias de formación memorables que necesitan ser interpretadas y puestas en clave de conocimiento didáctico y pedagógico para las generaciones de jóvenes que hoy se forman en el campo de la docencia en distintos lugares del mundo.

Debido a la relevancia de este tipo de investigación para la comprensión y generación de saber pedagógico y a la necesidad, cada vez más creciente en el ámbito de la investigación y formación docente, de conocer sus antecedentes, sus fundamentos conceptuales, las técnicas y estrategias asociadas, sus posibilidades de uso y los resultados de aplicación, en este artículo de revisión se presentan algunos antecedentes históricos de este método y los resultados de una exploración sistemática de literatura que puso en el centro de análisis la relación entre dos categorías temáticas: las investigaciones con objeto de estudio centrado en la identidad docente y con diseño metodológico basado en el método biográfico narrativo. Esto, con el fin de establecer las tendencias predominantes en la investigación de este campo de estudio.

La selección de la muestra documental se realizó a través de un rastreo en varias bases de datos: jstor, Bases de datos de Tesis Doctorales (TESEO), Dialnet, Tesis Doctorales en ReD (TDR), Google Scholar, REDIAL-TESIS, Portal de Tesis Latinoamericanas. Ellas permitieron encontrar un corpus de 15 tesis doctorales publicadas en el periodo 20042017 y de 37 artículos de investigación divulgados en el ciclo 2002-2016.

Metodológicamente se procedió con elementos de los estudios de metasíntesis, un ejercicio que redimensiona la búsqueda de información pertinente a un tema de interés para integrar las manifestaciones de investigación cualitativa, con el fin de lograr unas nuevas interpretaciones que explican el fenómeno con un nivel mayor de evidencias a través de síntesis interpretativas (Carreño, 2012). El objetivo general de esta propuesta es reconocer los alcances y principales procesos metodológicos de la investigación narrativa, además de contar con información para determinar vacíos en este campo de investigación.

El estudio de los textos escogidos se efectuó a través de fichas de revisión y análisis documental, teniendo en cuenta aspectos como objeto de estudio, objetivos, diseño metodológico y resultados. En cada uno de los anteriores rasgos se identificaron subcategorías con las cuales se establecieron diversas tendencias. Las corrientes identificadas se centran en la utilidad de la investigación narrativa, objetivos de las investigaciones, principales técnicas y estrategias de investigación, y los resultados y significación práctica de los estudios revisados.

\section{Antecedentes históricos de la investigación biográfico narrativa}

Este tipo de investigación se relaciona, en sus comienzos, con la construcción de historias de vida, las cuales fueron empleadas desde principios del siglo $\mathrm{xx}$ con fines sociológicos, antropológicos, feministas y sociolingüistas (Thomas y Znaniecki, 1920, 1930; Shawm 1930, 1966; Sutherland, 1937; Radin, 1926; Botkin, 1945; Personal Narrative Group, 1989; Lanov y Waletzky, 1967,1997, citados en Chase, 2015). En el ámbito sociológico, se emplearon los registros de vida para comprender las instituciones sociales y explicar la conducta del individuo como un proceso interactivo entre el sujeto y el medio sociocultural. En el contexto antropológico, posibilitaron registrar la cultura de grupos indígenas estadounidenses y establecer relaciones entre estas y los individuos. En la esfera feminista reflejaron las voces de esclavos estadounidenses y admitieron la creación de tensiones y transformaciones en los postulados androcéntricos de las ciencias sociales. En Sociolingüística, se reconoció a la narrativa como un discurso oral que merecía ser estudiado en sí mismo. 
Estos antecedentes confirman el uso de narrativas para comprender las estructuras sociales, el pensamiento de diversas poblaciones, su relación con el individuo, la correspondencia entre el modo de vida colectivo e individual, los movimientos institucionales y cambios sociales. En el caso específico de las historias de vida docente, Goodson (2004) acota que esta tendencia se empezó a desarrollar en el Reino Unido hacia los años 80 "comprendiendo la complejidad del docente como agente activo que construía su propia historia" (Goodson, 2004, p. 29). Se descubre, entonces, que entender la docencia, así como sus biografías y autobiografías, se conecta con la intención de centrar el conocimiento pedagógico en esta (Butt and Raymond, 1987, citado por Goodson, 2003), porque permite al docente hablar de sí mismo, de sus saberes de experiencia, mientras cuenta historias (Suárez, 2017).

Bolívar (2002) sitúa a la investigación biográfico narrativa como una perspectiva que comporta credibilidad y legitimidad propia para reconocer, reposicionar y construir conocimiento en el ámbito educativo, además de tener una identidad propia en la investigación (Bolívar y Domingo, 2006; Porta, 2010). A partir de este enfoque, las autobiografías docentes, las narrativas biográficas emergen como instrumento para comprender la identidad docente, los saberes pedagógicos asociados a esta y rescatar las identidades comunitarias. Sobre todo, en el contexto de la investigación iberoamericana, donde hay un rico campo para explorar objetos de estudio y metodologías que sustentan este planteamiento investigativo (Bolívar y Domingo, 2006).

\section{Concepciones sobre la utilidad de la investigación narrativa}

De acuerdo con Connelly y Clandinin (1995) la narrativa ha sido empleada con diversos sentidos: fenómeno que se investiga, método de investigación y estrategia de reflexión, cambio, innovación, mejora. En el caso del corpus documental revisado, son cinco las tendencias relacionadas con las concepciones sobre la utilidad y formas de uso de la investigación narrativa.

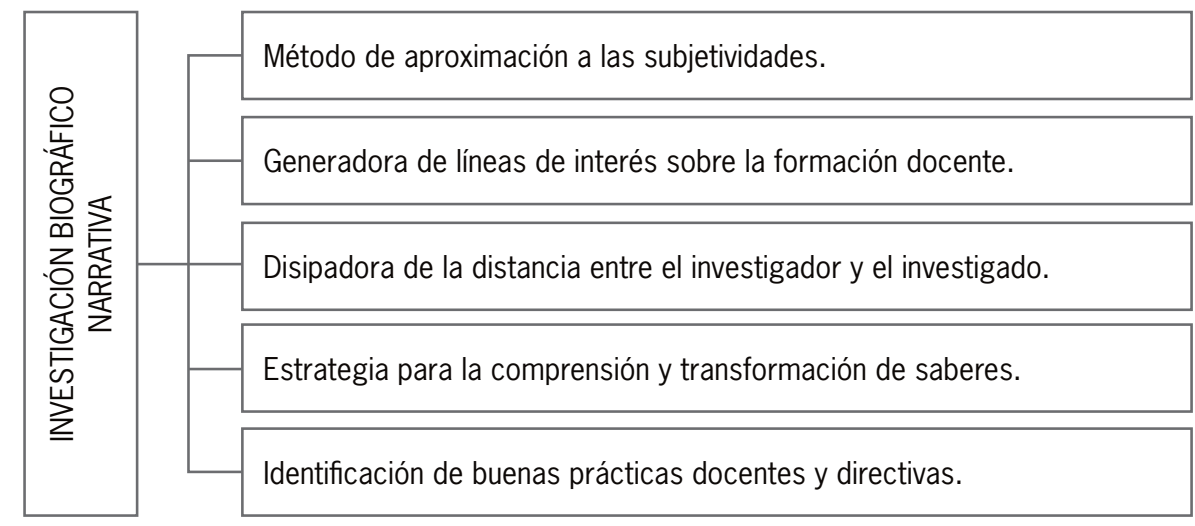

Gráfica 1. Concepciones sobre la utilidad de la investigación narrativa.

Fuente: elaboración propia.

Se plantea que el método biográfico narrativo posibilita la aproximación a las subjetividades y experiencias de los participantes de una investigación, mediante la reconstrucción de sus trayectorias vitales. Numerosos autores (González, 2004; Tsui, 2007; Ojeda, 2008; Sayago, Chacón y Rojas, 2008; Bolívar y
Bolívar, 2011; Oliveira, 2011; González, 2013; Cortés, Leite y Rivas, 2014; Delgado, 2015; Castañeda, 2016; García, 2016; Godino, 2017; Guzmán, 2017) consideran que este tipo de pesquisa se ha convertido en una forma de contarnos nuestra historia, ordenarla y estructurarla; de investigar, entender e interpretar el 
mundo subjetivo; de aproximarse a la vida cotidiana educativa de los profesores, explorar su vida singular; de recuperar las historias en relación con la identidad; de conocer la perspectiva individual- colectiva de los entrevistados; de construir la identidad profesional de los docentes y estudiantes de carreras en educación.

Se concibe no solo como método de investigación, sino como un enfoque o perspectiva en la que se usan las historias o relatos individuales para comprender a las personas dentro de su contexto social, sus identidades, subjetividades y emociones (López, 2003; Sparkes y Devís, 2007 citado en González, 2013). Las narrativas permiten a los docentes crear explicaciones sobre sí mismos, relacionadas con la disciplina que enseñan y su vida en función de esa disciplina (Lutovac y Kaasila, 2013). Lo plantea Guzmán la "investigación biográfico-narrativa representa una estrategia para entrar en el mundo de los significados y los saberes en claves cotidianas presentes en los procesos de interrelación, identificación y reconstrucción personal y cultural" (2017, p. 136).

La segunda tendencia, acerca de utilidad del método biográfico narrativo demuestra que esta metodología ha sido generadora de líneas de interés sobre la formación docente, sobre la vida y la experiencia profesional de los profesores. Diversos autores (Merino, 2015; García, 2016; Godino, 2017) la reconocen como un aporte para promover el desarrollo profesional e identificar el significado que los investigados le otorgan a la formación y a sus prácticas de enseñanza (Fernández, 2010; Godino, 2017). También, se presenta como enfoque sostenible para la transferencia de las buenas prácticas formativas y la mejora de las políticas respecto a la formación docente, producto de la posibilidad de acceso a las narrativas de profesores memorables (Fernández, 2012).

En tercer lugar, se razona sobre la influencia que ha tenido la investigación narrativa en estrechar el vínculo entre los investigadores y los sujetos de investigación, para reducir las diferencias de poder entre ellos (Norton y Early, 2011). Al respecto, García estipula que "este enfoque se sitúa y aborda el vínculo investigadora- investigada (...) e intenta difuminar la distancia entre ambas" (2016, p. 101).
En otro sentido, se afirma que la investigación biográfico narrativa promueve la comprensión y transformación de los saberes de quien narra las historias y la construcción de conocimiento en la investigación educativa y social, conlleva a la reflexión y la transformación de los saberes pedagógicos de la acción educativa. Se concluye que este método permite reflexionar sobre aspectos relevantes para el desempeño docente como sus trayectorias de vida, sus identidades, sus sentimientos, propósitos y deseos, y mientras narran los participantes escuchan a otros, se escuchan a sí mismos, integran la práctica pedagógica, los saberes disciplinares, los procesos investigativos del aula; lo cual lleva a experiencias transformadoras, mediante palabras generadoras que permiten analizar e interpretar las realidades educativas (Huchim y Reyes, 2013; González, 2016; García, 2016; Godino, 2017).

Según Merino (2015), presentar las experiencias bajo los cánones narrativos supone un modo de construir realidad, lo cual se ratifica con los planteamientos de Bruner, porque la narrativa no solo es un tipo de texto, sino un modo de pensamiento: "[...] los hablantes construyen acontecimientos valiéndose de la narrativa en vez de simplemente referirse a ellos" (Bruner, citado por Chase, 2015, p. 69). Se colige que cuando los docentes narran sus trayectorias de vida pueden reflexionar y construir pensamiento pedagógico, saber pedagógico: "los investigadores narrativos consideran la narrativa como la creación de significado en retrospectiva" (Chase, 2015, p. 69).

Por último, la identificación de las buenas prácticas docentes surge como una categoría dentro de la Investigación biográfico narrativa. Los relatos docentes brindan al investigador la posibilidad de identificar y conceptualizar acerca de las buenas prácticas de enseñanza (Álvarez y Porta, 2012), mediante el análisis de las prácticas pedagógicas de docentes reconocidos en la esfera educativa, principalmente en el ámbito universitario. Las significaciones emergen tanto de los relatos de los docentes memorables como del significado atribuido por sus estudiantes (Guzmán, 2017). En este contexto, un enfoque denominado por Porta y Yedaide (2013) “autobiográfico 
narrativo" ha permitido conceptualizar la pasión en los docentes universitarios. Al convertirse la metodología de la investigación narrativa en un ejercicio de realidades vividas de los maestros a partir de conversaciones sobre la realidad, la compresión no solo está en el sujeto sino en su práctica de profesión y de vida.

Además, Bolívar y Ritacco (2006) utilizan este enfoque para vincular la identidad profesional de los directivos con el liderazgo escolar exitoso.

\section{Objetivos de la investigación narrativa}

Se hallaron cuatro tendencias en los objetivos generales de las investigaciones biográfico-narrativas. Primero, las investigaciones asociadas con la construcción y perfilación de la identidad docente; segundo, las pesquisas que buscan una aproximación a la situación de la profesión docente; además, las indagaciones que reconocen y comprenden los saberes docentes; por último, las que estudian las buenas prácticas docentes.

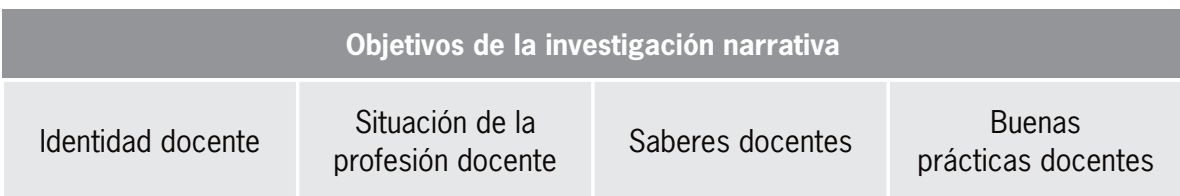

Gráfica 2. Objetivos de la investigación narrativa.

Fuente: elaboración propia.

Respecto de la identidad se advierte que el método en mención ha sido empleado para indagar en las experiencias, interpretaciones y significados asociados a la identidad docente (Sayago, Chacón y Rojas, 2008; García, 2010; Contreras, 2013; Vargas, 2013; Domínguez, Ruiz y Medina, 2017); construir otras lecturas sobre la identidad del docente de posgrados y comprenderla desde los planteamientos de la perspectiva de la complejidad (Castañeda, 2016); estudiar y comprender la identidad profesional del docente novel en sus primeros años de profesión, la del docente del nivel primario y del nivel secundario en el contexto de la sociedad global (González, 2013; Godino, 2017; Merino, 2015); conocer los contenidos y rasgos constitutivos de esta identidad en estudiantes de pedagogía (Guzmán, 2017); interpretar las conexiones, relacionamientos y entrecruzamientos entre los relatos de vida de maestras especiales y los procesos de reforma educativa a la educación especial (Yarza, Ramírez, Franco y Vásquez; 2014).

En cuanto a las indagaciones que realizan una aproximación a la situación de la profesión docente,
Delgado (2015) tuvo como objetivo reconstruir las historias de vida de docentes universitarios desde incidentes críticos, como las condiciones sociopolíticas, familiares y educativas que influyen en el desarrollo personal y profesional; González (2013), quiso comprender cómo la práctica reflexiva, a partir de vivencias y sentimientos personales permite examinar y reconstruir la enseñanza. Adicionalmente, entre las investigaciones que se centra en el reconocimiento y comprensión de saberes docentes están las que buscan comprender los saberes educativos desde la experiencia vivida y narrada de mujeres artistas docentes (García, 2016).

Cierran esta categoría las pesquisas que indagan sobre las buenas prácticas docentes o prácticas de enseñanza memorable. El trabajo de Álvarez, Porta y Sarasa (2010) exploró las biografías de una selección de buenos profesores. Mientras que en la investigación de Branda y Porta (2012) se analizó la historia de vida de un docente universitario, previamente identificado como docente memorable por los estudiantes. 


\section{Diseños metodológicos: principales técnicas y estrategias de investigación}

Como producto de la metasíntesis realizada se identificó el empleo recurrente de las siguientes técnicas y estrategias de investigación narrativa, que pueden ser orientadoras en la concepción y diseño de indagaciones de esta naturaleza.

\section{Técnicas de investigación}

Las investigaciones con enfoque biográfico narrativo se han basado en varias técnicas, entre ellas los relatos de experiencia, la entrevista semiestructurada, la entrevista a profundidad, los biogramas o autobiogramas y grupos de discusión. A continuación, se conceptualizan desde la perspectiva de las investigaciones analizadas.

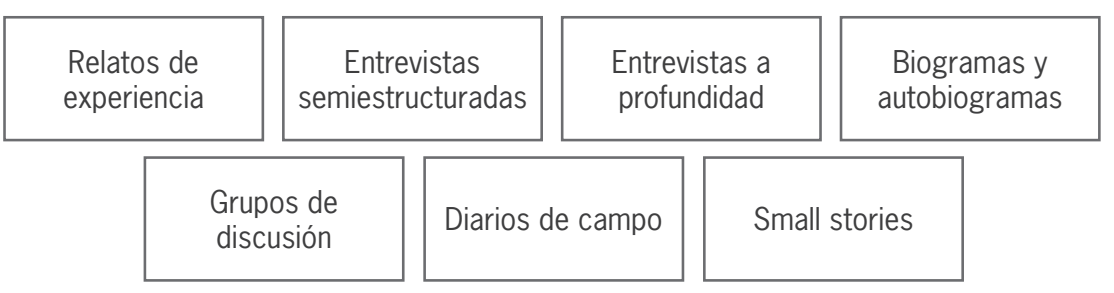

Gráfica 3. Técnicas y estrategias para la investigación biográfico narrativa.

Fuente: elaboración propia.

Los relatos de experiencia, relatos de vida o narrativas docentes son usados para identificar los incidentes críticos en la vida de los profesores (Castañeda, 2016). Constituyen un instrumento para reconstruir significantes de las trayectorias de vida y laboral a través de relatos centrados en aspectos específicos (Mortola, 2006; Yarza, Ramírez, Franco y Vásquez, 2014; Guzmán, 2017). Son textos únicos que se producen en torno a los docentes y a partir de preguntas o temas de interés de la vida de quienes narran.

Las entrevistas semiestructuradas han sido empleadas con sentido de encuentro, de comprensión, de escucha, de conocer más al otro; hablando sobre aspectos personales y profesionales, para que los docentes reconstruyan, de manera libre y espontánea, sus trayectorias (Mortola, 2006; González, 2013).

Las entrevistas a profundidad, consideradas por algunos investigadores como el principal instrumento de los estudios biográfico narrativos (Delgado, 2015; Guzmán, 2017), han permitido identificar experiencias familiares, escolares, de formación, de práctica docente, relación con el currículo, relación con los estudiantes. Se han desarrollado a través de conversaciones mediante la reflexividad, el pensamiento en voz alta y la estimulación del recuerdo en atención a aspectos como el contexto sociocultural, las percepciones y valoraciones de los entrevistados, respecto a sus funciones, capacidades, posiciones, limitaciones, etc. (Bolívar, Fernández y Molina, 2005; Mortola, 2006; Ojeda, 2008; Merino, 2015; Bolívar y Ritacco, 2016; García, 2016; Domínguez, Ruiz y Medina, 2017; Godino, 2017).

Los biogramas y autobiogramas son líneas de tiempo, mapas de las trayectorias personales y profesionales para estructurar, así como secuenciar cronológicamente los acontecimientos y experiencias vivenciadas por el profesorado (Delgado, 2015; Castañeda, 2016).

Los grupos de discusión son conversaciones cuidadosamente planeadas y diseñadas como espacio de encuentro y aprendizaje colectivo, con el objetivo de compartir experiencias y obtener información mediante la interacción del grupo sobre un tópico propuesto por el investigador (Bolívar, Fernández y Molina, 2005; Merino, 2015; García, 2016).

Los diarios de campo, cuadernos de campo o diarios de clase han sido empleados para registrar las circunstancias transcurridas en las sesiones de 
trabajo, como los datos significativos, las sensaciones y reflexiones del propio investigador (González, 2013; Delgado, 2015; Godino, 2017).

\section{Estrategias de investigación}

Small Stories: son una estrategia propuesta como antídoto a los estudios narrativos canónicos en la cual se emplean cuentos pequeños, narraciones de eventos en curso, eventos futuros o hipotéticos eventos compartidos para lograr la función de interacción en la que se espera que los participantes de la investigación manifiesten sus representaciones sobre los acontecimientos pasados, el sentido de sí mismos sobre estos acontecimientos (Norton y Early, 2011). En términos de Bamberg y Georgeakopoulos:

we have been employing 'small stories' as an umbrella-term that captures a gamut of under-represented narrative activities, such as tellings of ongoing events, future or hypothetical events, shared (known) events, but also allusions to (previous) tellings, deferrals of tellings, and refusals to tell. $(2008$, p. 5$)$

\section{Resultados y significación práctica de los estudios realizados}

Las distintas investigaciones revisadas dan cuenta de hallazgos fundamentales en relación con identidad docente, saber pedagógico, docentes memorables y riqueza de la investigación narrativa.

\section{Identidad docente}

Se concluye que entre principios epistemológicos para comprender el concepto de identidad docente desde la perspectiva compleja se encuentran: el proceso identitario requiere de miradas integradoras sobre los contextos que rodean al maestro; la narraciones sobre la historias propias permiten que surjan nuevas compresiones de sí; los procesos reflexivos que se dan a través de las narrativas permiten al docente su autoconstrucción; en la construcción de la identidad docente intervienen encuentros entre los sistemas macro y micro de las historias de vida (Castañeda, 2016).
En otro orden de ideas, la identidad docente es múltiple y dinámica (Sayago, Chacón y Rojas, 2008; Guzmán, 2017), es decir, un educador en formación tiene diversas representaciones identitarias sobre los roles y funciones de la profesión: educador, experto en el diseño, didáctica, metodología y en el contenido disciplinario. Se plantea necesario resignificar el enfoque de formación de la identidad profesional, incorporando acciones formativas relacionadas con esta multiplicidad (Guzmán, 2017).

El proceso de construcción de la identidad profesional docente está ligado y condicionado por su identidad personal, por lo cual se debe tener una visión global de los profesores (González, 2013). En el caso de los futuros profesores las posibilidades de identidades decisivas e irresolutas (Lutovac y Kaasila, 2013), relacionadas con la existencia de objetivos claros y autoconfianza para ejercer la profesión docente, llevan a considerar las narrativas como metodología de formación (Coulter, Michael y Poynor, 2007) orientada hacia la construcción de una identidad profesional sólida que permita un exitoso desempeño profesional.

La identidad del profesor evoluciona a partir de los procesos conflictivos generados entre la formación inicial de la educación primaria y secundaria, el paso por la universidad y las primeras experiencias profesionales en las escuelas. A través del conocimiento que adquiere en la reflexión sobre su experiencia y a través de la comprensión de la docencia como trabajo colectivo mediante el encuentro entre pares (González, 2013; Cortés, Leite y Rivas, 2014; Godino, 2017; Gómez, 2015).

En la identidad profesional de los profesores de secundaria, en el contexto de una sociedad global, se identificaron problemas debido a las nuevas y difíciles condiciones laborales, al escaso reconocimiento o indiferencia social, a la desvalorización del profesor, aspectos que podrían influir en la pérdida de entusiasmo por la profesión, la disminución de la autoestima y la desestabilización de la autoimagen (Bolívar, Fernández y Molina, 2005; Vaillant, 2008; Bolívar, 2011; Merino, 2015). Se reconoce un desafecto generalizado y debilitamiento del sentido de pertenencia profesional cuando se atribuyen al 
docente nuevas funciones de socialización en el contexto educativo contemporáneo (Iannaccone, Tateo, Mollo y Marsico, 2008).

\section{Saber pedagógico}

Se ha concluido que el saber docente no está determinado por una posición de sujeto individual, las experiencias vividas junto a otros, que se articulan socialmente, también la determinan: es una identidad intersubjetiva, ligada a las relaciones con los demás. Así, una mirada integradora de experiencias complejas define el ser docente. (González, 2013; García, 2016). Los estudios confirman la característica relacional de la identidad docente, en la que la relación con los estudiantes y las relaciones profesionales cobran importancia (Guzmán, 2017).

La investigación narrativa contribuye a la realización de prácticas docentes reflexivas, que convierten a los profesores en generadores de conocimiento (González, 2013).

\section{Los rasgos de un docente memorable}

Se concluye que el valor dado a las experiencias personales y profesionales, la formación disciplinar e interdisciplinar, la reflexión sobre el ejercicio profesional y la internacionalización de las labores de gestión académica son centrales en el éxito de los mejores docentes universitarios (Delgado, 2015).

Además, hay una estrecha relación entre lo que los alumnos y docentes consideran un ejemplo de buena enseñanza por “[...] la relación especular que a menudo se entabla entre los modelos docentes y los modelos de los estudiantes, y la influencia de la biografía escolar en sus concepciones sobre la buena enseñanza” (Álvarez y Porta, 2012, p. 86).

Finalmente, en los relatos de profesores reconocidos por sus buenas prácticas de enseñanza es recurrente la alusión a términos relacionados con la pasión. Lo cual lleva a considerar la necesidad, de acuerdo con los investigadores, de incluir esta categoría en el currículo de la formación docente (Porta y Yedaide, 2013).

\section{Riqueza de la investigación narrativa}

Los docentes resignifican su propia experiencia personal y profesional a través de este tipo de investigación, le otorgan un sentido mediante la interacción que subyace en la investigación biográfico narrativa, articulan las dimensiones individual y colectiva construidas históricamente en los procesos sociales. Esto, porque el relato personal necesariamente provoca la reflexión personal y colectiva (Bolívar, Fernández y Molina, 2005; Álvarez y Porta, 2012; Cortés, Leite y Rivas, 2014).

Además, constituye un gran potencial formativo para que los docentes en formación analicen las creencias, conocimientos, imágenes y representaciones adquiridas biográficamente (Mortola, 2006). $\mathrm{Y}$ se ha convertido en fuente de nuevas formas de comprensión sobre los procesos relacionados con la docencia (Porta y Yedaide, 2014).

\section{Conclusiones}

La emergencia del campo de conocimiento se dio entre la década entre 1970 y 1980 en medio de una fuerte incursión de los estudios fenomenológicos-humanísticos. Lo que muchos autores denominaron "géneros borrosos" que se atrevieron a incursionar una diversidad de paradigmas, teorías, métodos y estrategias de investigación, que incluyen una audaz crítica al positivismo, pero especialmente se logró admitir que la escritura podría ser un método de investigación por fuera de la presentación de resultados.

En atención a la consulta realizada se tiene que, metodológicamente, la investigación narrativa por su naturaleza no se configura con fronteras rígidamente definidas con instrumentos preestablecidos y validados con rigores estandarizados; sin embargo, la narración no es un vínculo sino un todo de sentido, lo que implica que existe una continua exploración de métodos y de instrumentos en su sentido espiral que podrían desarrollarse aún más.

Existe además una fuerte tendencia descalificadora de los estudios narrativos-cualitativos, con que los acusan de consolidarse con proyectivas disciplinarias hacia la literatura que consideran extensa 
en historias y limitada en métodos. Estas miradas no consideran la naturaleza del sentido dentro de la investigación y las posibilidades de construcción de significado que se han mostrado a lo largo de esta metasíntesis.

Así entonces, la narrativa utilizada para la compresión de las prácticas docentes puede definirse, tal como lo plantean Arias y Alvarado como:

una historia que les permite a las personas dar sentido a sus vidas. Consiste en un esfuerzo del sujeto por conectar su pasado, su presente y su futuro de tal manera que se genere una historia lineal y coherente consigo misma y con el contexto. (2015, p. 172)

Este enfoque de investigación ha permitido desarrollar conocimiento sobre la formación docente, sobre la vida y la experiencia profesional de los profesores, sobre cómo las condiciones sociopolíticas, familiares y educativas influyen en el desarrollo personal y profesional, acerca de las concepciones sobre la buena enseñanza, la identidad docente, el saber pedagógico, los rasgos de los docentes memorables y la riqueza de la investigación narrativa.

\section{Referencias}

Álvarez, Z. y Porta, L. (2012). Caminos de indagación sobre la buena enseñanza: aproximación biográfico-narrativa en educación superior. Revista de Educación, (4), 75-88.

Álvarez, Z., Porta, L. y Sarasa, M. (2010). Itinerarios de la buena enseñanza a partir de los relatos biográficos docentes. Profesorado. Revista de Currículum y Formación de Profesorado, 14(3), 89-98.

Arias, A. y Alvarado, S. (2015). Investigación narrativa: apuesta metodológica para la construcción social de conocimientos científicos. Revista CES Psiología, 8(2), 171-181.

Bamberg, M. y Georgakopoulou, A. (2008). Small Stories as a New Perspective in Narrative and Identity Analysis. Text \& Talk, 28(3), 377-396. https://doi. org/10.1515/TEXT.2008.018

Bolívar, A. (2011). La investigación biográfica narrativa en el desarrollo e identidad profesional del profesorado. En J. González, Les histoires de vie en Espagne: entre formation, identité et mémoire (págs. 59-96). L'Harmattan.
Bolívar, A. y Bolívar, R. (2011). La didáctica en el núcleo del mejoramiento de los aprendizajes. Entre la agenda clásica y actual de la didáctica. Revista Perspectiva Educacional, 50(2), 3-26.

Bolívar, A. y Domingo, J. (2006). La investigación biográfica y narrativa en Iberoamérica: Campos de desarrollo y estado actual. Forum Qualitative Social Research, 7(4), 1-33.

Bolívar, A. y Ritaco, M. (2016). Identidad profesional de los directores escolares en España. Un enfoque biográfico narrativo. Revista de Ciencias Humanas y Sociales, (79), 163-183.

Bolívar, A., Fernández, M. y Molina, E. (2005). Investigar la identidad profesional del profesorado: Una triangulación secuencial. Forum Qualitative Social Research, 6(1), 1-26.

Bolívar, B. (2002). “De nobis ipsis silemus?”: Epistemología de la investigación biográfico-narrativa en educación. Revista Electrónica de Investigación Educativa, 4(1).

Branda, S. y Porta, L. (2012). Maestros que marcan. Biografía e indentidad profesional en docentes memorales. Profesorado. Revista de curriculum y formación del profesorado, 16(3), 231-243.

Bruner, J. (1998). Realidad mental y mundos posibles. Gedisa.

Carreño, S. (2012). Metasíntesis de calidad de vida en cuidadores familiares de personas en situación de enfermedad crónica. [Tesis de maestría, Universidad Nacional de Colombia]. Repositorio institucional un. http://www.bdigital.unal.edu.co/8564/

Castañeda, A. (2016). Identidad del docente de posgrados en educación. Una mirada compleja. [Tesis de doctorado, Universidad Santo Tomás]. Repositorio institucional UsTa. https://repository.usta.edu.co/ bitstream/handle/11634/2987/Castanedaana2016. pdf? sequence $=1$ \&isAllowed $=y$

Chase, S. (2015). Investigación narrativa. Multiplicidad de enfoques, perespectivas y voces. En N. Denzin y $\mathrm{Y}$. Lincoln, Métodos de recolección y análisis de datos (pp. 58-112). Gedisa.

Connelly, M. y Clandinin, J. (1995). Relatos de experiencia e investigación narrativa. En J. Larrosa, Déjame que te cuente. Ensayos sobre narrativa y educación (pp. 11-59). Laertes.

Contreras, C. (2013). La formación del profesorado universitario orientada hacia la trasnformación de la identidad docente. Una propuesta basada en incidentes críticos. Universitat Autónoma de Barcelona. 
Cortés, P., Leite, A. y Rivas, J. (2014). Un enfoque narrativo de la identidad profesional en profesorado novel. Tendencias pedagógicas, (24), 199-214.

Coulter, C., Michael, C. y Poynor, L. (2007). Storytelling as Pedagogy: An Unexpected Outcome of Narrative Inquiry. Curriculum Inquiry, 37(2), 103-122.

Delgado, M. (2015). Historias de vida profesional docente y tutoría en la universidad. Universidad de Huelva.

Domínguez, M., Ruiz, A. y Medina, A. (2017). Experiencias docentes y su proyección en la identidad profesional: el caso de las maestras de Santa Marta y su entorno. Revista historia de la educación latinoamericana, 19(29), 111-134. https://doi. org/10.19053/01227238.7555

Fernández, M. (2010). Aproximación biográfico-narrativa a la investigación sobre formación docente. Profesorado. Revista de Currículum y Formación de Profesorado, 14(3), 17-32.

Fernández, M. (2012). Aportes de la aproximación biográfico-narrativa al desarrollo de la formación y la investigación sobre formación docente. Revista de educación, (4), 11-36.

García, M. (2010). La identidad docente: constantes y desafíos. Revista Interamericana de Investigación, Educación y Pedagogía, 3(1), 15- 42. https://doi. org/10.15332/s1657-107X.2010.0001.01

García, R. (2016). Diálogos, desplazamientos y experiencias del saber pedagógico. [Tesis de doctorado, Universitat de Barcelona]. Repositorio institucional UB. http://hdl.handle.net/2445/102787

Godino, C. (2017). Procesos de construcción de nuevas identidades docente. Vinculaciones entre los mandatos de formación inicial y los contextos de trabajo profesional en las instituciones de nivel primario. [Tesis de doctorado, Universidad de Buenos Aires]. Repositorio institucional UBA. http://repositorio. filo.uba.ar/jspui/bitstream/filodigital/4365/1/ uba_ffyl_t_2017_se_godino_v1.pdf

Gómez, F. (2015). La identidad profesional de los profesores de matemáticas y ciencias sociales en la educación secundaria. [Tesis de doctorado, Universidad Autónoma de Barcelona]. Repositorio institucinal UAB. https://ddd.uab.cat/pub/tesis/2016/ hdl_10803_377472/fgt1de1.pdf

González, A. (2004). Poder y discursos en la construcción social de las identidades docentes universitarias. [Tesis de doctorado, Universitat de Valencia].
González, G. (2013). Evolución de la identidad profesional de un docente novel de educación físsica: anaálisis a partir de la reflexión de sus experiencias personales y de su propia práctica. [Tesis de doctorado, Universidad de Valladolid]. Repositorio institucional uva. http://uvadoc.uva.es/handle/10324/2817

González, H. (2016). Caracterización del saber pedagógico de los profesores del proyecto académico de investigación y extensión de pedagogía - PAIPEP- en la Universidad Distrital Francisco José de Caldas. [Tesis de doctorado, Universidad de Manizales].

Goodson, I. (2003). Hacia un desarrollo de las historias personales y profesionales de los docentes. Revista Mexicana de Investigación Educativa, 8(19), 733-758.

Goodson, I. (2004). Historias de vida del profesorado. Ediciones Octaedro, S.L.

Guzmán, L. (2017). La construcción de la identidad profesional docente. Estudio cualitativo sobre la construcción de la identidad profesional de los estudiantes de pedagogía den programas de formación inicial de profesores de carácter público y privado. [Tesis de doctorado, Universitat de Girona].

Huchim, D. y Reyes, R. (2013). La investigación biográfico- narrativa, una alternativa para el estudio de los docentes. Revista Electrónica "Actualidades Investigativas en Educación”, 13(3), 1-27.

Iannaccone, A., Tateo, L., Mollo, M. y Marsico, G. (2008). L'identité professionnelle des enseignants face au changement : analyses empiriques dans le contexte italien. Travail et formation en éducation, (49), 1-26.

López, S. (2003). Construcción sociocultural de la profesionalidad docente: estudio de casos de profesores comprometidos con un proyecto educativo. [Tesis de doctorado, Universitat de Valencia].

Lutovac, S. y Kaasila, R. (2013). Pre-service tcachcrs' future-oriented mathematical identity work. Springer Science+Business Media Dordrecht, (85), 129- 142. https://doi.org/10.1007/s10649-013-9500-8

Merino, M. (2015). El desarrollo de la identidad docente en el profesorado de educación secundaria en un nuevo contexto social. Un enfoque Biográfico- Narrativo. [Tesis de doctorado, Universidad de Sevilla]. Repositorio institucional us. http://hdl.handle. net/11441/34717

Mortola, G. (2006). Una aproximación narrativa a la construcción de la identidad laboral docente. Educación, Lenguaje y Sociedad, 4(4), 83-104. 
Norton, B. y Early, M. (2011). Researc her Identity, Narrative Inquiry, and Language Teaching Research. TESOL Quarterly, 45(3), 415-439.

Ojeda, M. (2008). Rasgos de la identidad del profesor de enseñanza media en su trayectoria de formación y desempeño profesionales. ¿Cómo, cuándo y con quiénes adquiere su condición de profesor? Revista Electrónica de Investigación Educativa, 10(2), 2-14.

Oliveira, A. (2011). A importância da investigação narrativa na educação. Educ. Soc., Campinas, 32(114), 171-188.

Porta, L. (2010). La investigación biográfico narrativa en educación. Entrevista a Antonio Bolívar. Revista de Educación, (1), 201-212.

Porta, L. y Yedaide, M. (2013). La pasión educa: enunciaciones apasionadas de profesores memorables universitarios. Revista Argentina de Educación Superior, (6), 35-50.

Porta, L. y Yedaide, M. (2014). La investigación biográfico narrativa. Desafíos ontológicos para la investigación y la enseñanza en la formación de formadores. Sophia, Colección de Filosofía de la Educación, (17), 177-192. https://doi.org/10.17163/ soph.n17.2014.22
Sayago, Z., Chacón, M. y Rojas, M. (2008). Construcción de la identidad profesional docente en estudiantes universitarios. Investigación arbitrada, 12(42), 551-561.

Suárez, D. (2017). Relatar la experiencia docente. La documentación narrativa del mundo escolar. Revista Teias, 18(50), 193-209.

Tsui, A. (2007). Complexities of Identity Formation: A Narrative Inquiry of an EFL Teacher. TESOL Quarterly, 41(4), 657-680. https://doi.org/10.1002/ j.1545-7249.2007.tb00098.x

Vaillant, D. (2008). La Identidad Docente. Importancia del Profesorado. Revista Investigaciones en Educación, 8(1), 15-38.

Vargas, A. (2013). Revisión entre iguales, escritura académica e identidad en la formación docente en una universidad colombiana. [Tesis de doctorado, Universitat Pompeu Fabra].

Yarza, A., Ramírez, M., Franco, L. y Vásquez, N. (2014). Reformas, Relatos de Vida e Identidades Profesionales en Educación Especial: una Aproximación a Partir de las Voces de Educadores Especiales en Medellín (Colombia), 1965-2002. Revista Brasileira de Educação Especial, 20(2) 325-340. http://dx.doi. org/10.1590/S1413-65382014000300002 